\title{
Altered and Agitated due to Hypertension: A Case of Posterior Reversible Encephalopathy Syndrome
}

\author{
Raza Kazmi $^{1}$, Emily Clark ${ }^{1}$, Vir Singh ${ }^{2,3}$, Michael Falgiani ${ }^{1}$, Latha Ganti ${ }^{4,5,} 6$ \\ 1. Emergency Medicine, Ocala Regional Medical Center, University of Central Florida College of Medicine, Ocala, USA \\ 2. Emergency Medicine, University of Central Florida College of Medicine, Orlando, USA 3. Emergency Medicine, Ocala \\ Regional Medical Center, Ocala, USA 4. Emergency Medicine, Envision Physician Services, Nashville, USA 5. Emergency \\ Medicine, University of Central Florida College of Medicine/Hospital Corporation of America Graduate Medical \\ Education Consortium of Greater Orlando, Orlando, USA 6. Emergency Medical Services, Polk County Fire Rescue, \\ Bartow, USA
}

Corresponding author: Latha Ganti, latha.ganti@ucf.edu

\section{Abstract}

The authors present a case of posterior reversible encephalopathy syndrome in a patient on nutritional supplements. The presentation and emergency management are discussed.

Categories: Emergency Medicine

Keywords: posterior reversible encephalopathy syndrome (pres)

\section{Introduction}

Posterior reversible encephalopathy syndrome (PRES), as the name suggests, is a constellation of symptoms caused by reversible ischemia most commonly of the posterior cerebral vasculature [1]. Various terminologies have been used to describe this condition, including "reversible posterior leukoencephalopathy syndrome" and "reversible posterior cerebral edema syndrome" among others [2].

\section{Case Presentation}

A 55-year-old male presented to the emergency department (ED) via emergency medical services (EMS) with a chief complaint of altered mental status, seizure-like activity, and agitation.

The history of presenting illness and other information was obtained from EMS and the patient's wife, as the patient was agitated on arrival to the ED. According to the patient's wife, prior to arrival the patient had been complaining of the "worst headache of his life" after sexual intercourse, associated with vision changes and neck pain. He then suddenly began to have seizure-like activity, which his wife described as full body spasms. Immediately after this episode, the patient became altered. EMS stated that when they arrived at the home the patient was found to be sitting in a chair, appeared to be postictal, and then quickly became combative.

Received 05/28/2020

Review began 06/18/2020

Review ended 06/18/2020

Published 06/24/2020

\section{(c) Copyright 2020}

Kazmi et al. This is an open access article

distributed under the terms of the

Creative Commons Attribution License

CC-BY 4.0., which permits unrestricted
The patient had no significant past medical history, including no prior seizures. He endorsed similar headaches after intercourse over the last 10 years. The patient occasionally smoked marijuana and had a longstanding history of taking two over-the-counter nutritional supplements, according to the label recommendations (Table 1 ).

\begin{tabular}{|c|c|c|}
\hline $\begin{array}{l}\text { Name of } \\
\text { supplement }\end{array}$ & Ingredients & $\begin{array}{l}\text { Serving } \\
\text { size }\end{array}$ \\
\hline $\begin{array}{l}\text { Ultra Fast } \\
\text { Keto Boost }\end{array}$ & $\begin{array}{l}62 \mathrm{mg} \text { calcium beta hydroxybutyrate, } 32 \mathrm{mg} \text { magnesium beta hydroxybutyrate, } 10 \mathrm{mg} \text { sodium beta } \\
\text { hydroxybutyrate. Above as components of an } 800 \mathrm{mg} \text { "Go BHBC Proprietary Blend". Other ingredients } \\
\text { included gelatin, rice flour, stearate, and silicon dioxide. }\end{array}$ & $\begin{array}{l}\text { Two } \\
\text { capsules } \\
\text { daily }\end{array}$ \\
\hline $\begin{array}{l}\text { Clear Nails } \\
\text { Plus Extra } \\
\text { Strength } \\
\text { Nail } \\
\text { Formula }\end{array}$ & $\begin{array}{l}200 \text { mg/6 billion CFU “probiotic fungus blend”: Lactobacillus rhamnosus, Lactobacillus plantarum, } \\
\text { Bifidobacterium longum, Lactobacillus acidophilus, Lactobacillus salivarius, Bifidobacterium lactis, } \\
\text { Bifidobacterium bifidum, Lactobacillus fermentum, Lactobacillus reuteri, Inulin. } 450 \text { mg of turmeric and } 2.5 \\
\text { mg "BioPerine }{ }^{\mathrm{TM}} \text {, a proprietary piperine derivative Other ingredients include gelatin, maltodextrin, medium } \\
\text { chain triglycerides, magnesium stearate, and rice flour. }\end{array}$ & $\begin{array}{l}\text { One } \\
\text { capsule } \\
\text { daily }\end{array}$ \\
\hline
\end{tabular}

TABLE 1: The patient's nutritional supplements details 
A complete review of systems was unobtainable secondary to the patient's condition. Vital signs obtained included a pulse of 138 beats per minute, a blood pressure of $200 / 125 \mathrm{mmHg}$, and a blood glucose of 138 $\mathrm{mg} / \mathrm{dL}$. He remained combative, requiring medics to hold down each of his extremities to allow for emergent evaluation. Other than tachycardia and psychomotor agitation, the patient's physical exam was grossly normal. A comprehensive neurologic assessment was unable to be performed prior to sedation due to agitation. The patient was subsequently intubated for the safety of the patient and staff, and to allow for a complete emergency diagnostic workup to be performed. Rocuronium and etomidate were utilized for intubation, and the post-intubation sedation was achieved with propofol.

Differential diagnosis included meningitis, encephalitis, aspiration pneumonia, sepsis, subarachnoid hemorrhage, subdural hemorrhage, undiagnosed seizure disorder, and drug intoxication. The initial diagnostic workup included a complete blood count (CBC), a comprehensive metabolic panel (CMP), a lactic acid level, an arterial blood gas (ABG), a urine drug screen, ethanol, acetaminophen, and salicylate levels. Imaging included CT of the brain and CT angiography (CTA) of the brain, neck, and chest.

Significant laboratory findings included an arterial $\mathrm{pH}$ of $7.27, \mathrm{HCO}_{3}$ of $18.9 \mathrm{mEq} / \mathrm{L}$ with a base excess of -8 at an $\mathrm{FiO}_{2} 60 \%$, lactic acid of $5.95 \mathrm{mmol} / \mathrm{L}$, basic metabolic profile significant for carbon dioxide level $\left(\mathrm{CO}_{2}\right)$ of $11 \mathrm{mmol} / \mathrm{L}$, white blood cell (WBC) count of $18.9 \times 10^{3} / \mathrm{mm}^{3}$ with neutrophil count of $12.3 \%$. Toxicological screen was positive for tetrahydrocannibinol. Non-contrast CT of the brain, CTA of the head and neck, and CT brain perfusion were all without abnormal finding. CTA of the chest was significant for bibasilar pneumonia, which may have reflected aspiration.

The patient was subsequently admitted to the intensive care unit (ICU). In the ICU, the patient was hypotensive upon arrival. Sedation was discontinued, a central venous catheter was placed, and vasopressor support was initiated with norepinephrine. An electroencephalogram (EEG) was performed and did not show any epileptic spike and wave morphology. MRI of the brain was subsequently obtained, which showed parietal and occipital changes consistent with PRES (Figure 1). These radiologic findings and diagnosis could be clinically correlated with the patient's poorly controlled hypertension. 


\section{Cureus}

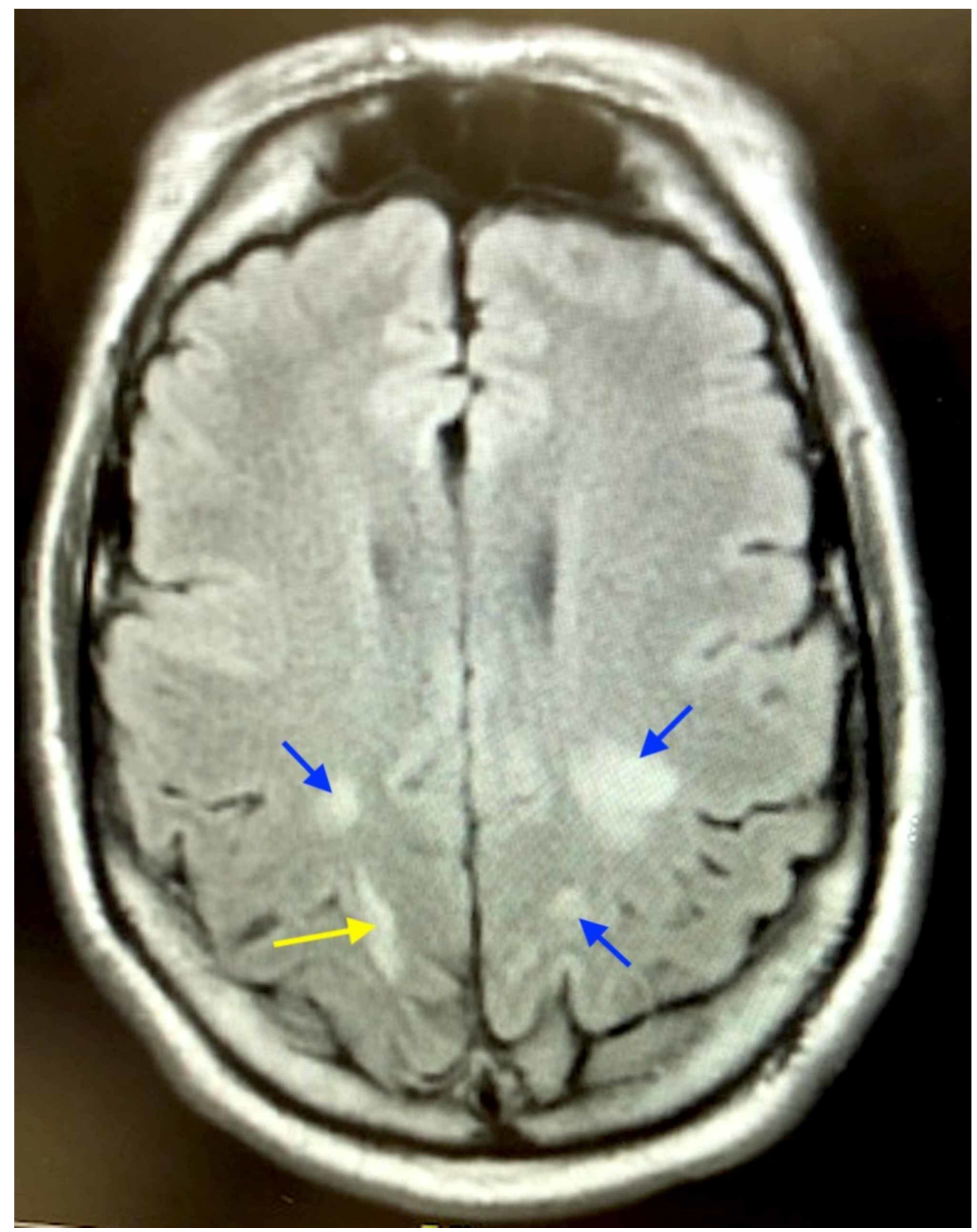

FIGURE 1: MRI demonstrating bilateral parietal and occipital subcortical T2 hyperintensities (blue arrows) with right occipital artifactual restricted diffusion (yellow arrow). No evidence of acute infarct, hemorrhage, hydrocephalus, or mass.

His hypertensive state in the ICU was managed originally with intravenous (IV) labetalol boluses, and subsequently with a nicardipine infusion. Piperacillin/tazobactam and azithromycin were administered for pneumonia, which were later switched to vancomycin and cefepime. The patient was extubated on inpatient day 2 and downgraded to the neurology floor on day 3. Neurology established a target blood pressure goal of systolic blood pressure below $140 \mathrm{mmHg}$. The patient was advised about the importance of blood pressure monitoring at home and connected with a primary care physician with whom to follow up within one week of discharge. He was discharged home on daily lisinopril.

\section{Discussion}

PRES is a neurological syndrome that usually evolves over hours, and typically presents with seizures, disturbed vision, headache, altered mental status, and encephalopathy accompanied by markedly elevated blood pressure [3]. PRES often develops in patients who are on cytotoxic medications, or in the context of renal disease, sepsis, or autoimmune disorders.

There are currently two theories as to the cause of PRES. One theory is that rapidly increasing blood pressure in hypertensive urgency or emergency exceeds the upper autoregulatory limits of cerebral 


\section{Cureus}

perfusion leading to vasogenic edema and leakage from cerebral vasculature. However, not all patients with PRES have elevated blood pressures at symptom onset, and approximately $30 \%$ of patients have normal or only slightly elevated pressures [2]. The second etiologic theory for PRES is that it is triggered by endothelial dysfunction due to circulating toxins. This theory is derived from the observation that PRES often occurs in patients who are undergoing treatment with cytotoxic medications, and have sepsis or renal disease. Circulating toxins in these disease states could alter the integrity of the vasculature of the cerebral circulation allowing for vasogenic edema and vascular leakage.

Suggested diagnostic criteria include acute onset of neurological symptoms, focal posterior vasogenic edema on imaging, and reversibility of clinical and/or imaging findings [4]. MRI classically shows evidence of symmetrical vasogenic edema in the posterior regions, often the parieto-occipital area. The common MRI features seen in PRES are summarized in Table $2[5,6]$.

\section{MRI findings in PRES}

Parieto-occipital dominance

Most often bilateral, and symmetrical

Vasogenic cerebral edema

Holohemispherical watershed distribution

Superior frontal sulcus

Subcortical white matter

Hyperintense T2-weighted and FLAIR sequences

DWI usually normal

Increased ADC values reflective of vasogenic cerebral edema

\section{TABLE 2: MRI findings in PRES}

ADC, apparent diffusion coefficient; DWI, diffusion-weighted imaging; FLAIR, fluid-attenuated inversion recovery; PRES, posterior reversible encephalopathy syndrome

Treatment of PRES is directed by symptoms and the underlying condition. Hypertension guidelines follow those of hypertensive emergency, and often seizure prophylaxis is warranted. When possible, treatment is aimed at removing underlying pathology or inciting factor. The patient outcomes are based on their underlying condition as PRES symptoms, and lesions seen on imaging are often reversible.

In our patient case, we found acute onset encephalopathy, which is a common presentation of PRES. He also had profound hypertension with the worst headache he had ever experienced which also follows with PRES syndrome. Our case is also the first documented case of PRES associated with the use of marijuana, keto, and antifungal supplements. Although use of these substances may be unrelated, it is important to document such associations in the event that a correlation may be established in the future. Thus, in our case, profound hypertension likely caused vasogenic edema and vascular leakage leading to PRES, which was further supported by posterior edema seen on MRI obtained during the hospital course.

\section{Conclusions}

It is imperative for emergency physicians to know about the clinical symptoms and radiological findings, diagnosis, and treatment of patients with PRES to initiate symptomatic and preventative treatment early in the disease process to possibly reduce complications and abbreviate syndrome duration.

\section{Additional Information}

\section{Disclosures}

Human subjects: Consent was obtained by all participants in this study. HCA Centralized Algorithms for Research Rules on IRB Exemptions (CARRIE)/ IRB manager issued approval 2020-374. Based on the information provided and attested as true, the research plan described does not require IRB oversight. This is because you are either a) not engaging in research with human subjects as defined by federal regulations; b) engaging in research with human subjects deemed excluded from IRB oversight per 45CFR46.102(l) OR c) engaging in research with sufficient human subject protections in the design to meet one or more IRB exemption criteria set forth in 45CFR46.104. Conflicts of interest: In compliance with the ICMJE uniform 


\section{Cureus}

disclosure form, all authors declare the following: Payment/services info: All authors have declared that no financial support was received from any organization for the submitted work. Financial relationships: All authors have declared that they have no financial relationships at present or within the previous three years with any organizations that might have an interest in the submitted work. Other relationships: All authors have declared that there are no other relationships or activities that could appear to have influenced the submitted work.

\section{Acknowledgements}

This research was supported (in whole or in part) by HCA Healthcare and/or an HCA Healthcare affiliated entity. The views expressed in this publication represent those of the author(s) and do not necessarily represent the official views of HCA Healthcare or any of its affiliated entities.

\section{References}

1. Hedna VS, Stead LG, Bidari S, et al.: Posterior reversible encephalopathy syndrome (PRES) and CT perfusion changes. Int J Emerg Med. 2012, 5:12. 10.1186/1865-1380-5-12

2. Feske SK: Posterior reversible encephalopathy syndrome: a review . Semin Neurol. 2011, 31:202-215. 10.1055/s-0031-1277990

3. Fischer M, Schmutzhard E: Posterior reversible encephalopathy syndrome. J Neurol. 2017, 264:1608-1616. 10.1007/s00415-016-8377-8

4. Fugate JE, Claassen DO, Cloft HJ, Kallmes DF, Kozak OS, Rabinstein AA: Posterior reversible encephalopathy syndrome: associated clinical and radiologic findings. Mayo Clin Proc. 2010, 85:427-432. 10.4065/mcp.2009.0590

5. Hinduja A: Posterior reversible encephalopathy syndrome: clinical features and outcome . Front Neurol. 2020, 11:71. 10.3389/fneur.2020.00071

6. Radiopeadia: Posterior reversible encephalopathy syndrome. (2020). Accessed: June 18, 2020: https://radiopaedia.org/articles/posterior-reversible-encephalopathy-syndrome-1?lang=us. 